\title{
Increased Expression of Both Constitutive and Inducible Forms of Nitric Oxide Synthase in the Delayed Phase of Acute Experimental Testicular Torsion
}

\author{
Changjong MOON ${ }^{1}$, Meejung $\mathrm{AHN}^{1)}$, Seungjoon $\mathrm{KIM}^{1)}$, Fumioki YASUZUMI ${ }^{2)}$ and Taekyun $\mathrm{SHIN}^{1) *}$ \\ ${ }^{1)}$ Department of Veterinary Medicine, Cheju National University, 1 Ara-1-dong, Jeju 690-756, South Korea and ${ }^{2)}$ Unit of Physiological \\ Science Anatomy 2, Faculty of Medicine, University of the Ryukyus, 207 Uehara, Nishihara-cho, Okinawa 903-0215, Japan
}

(Received 3 September 2004/Accepted 24 November 2004)

ABSTRACT. To elucidate the roles of both constitutive endothelial nitric oxide synthase (eNOS) and neuronal NOS (nNOS), and inducible NOS (iNOS) in acute experimental testicular torsion, the expression of iNOS and constitutive eNOS and nNOS were studied in the rat testis with ischemia/reperfusion (I/R) injury. Western blot analysis showed that all three isoforms of NOS increased significantly at 24 $48 \mathrm{hr}$ after I/R and declined slightly thereafter. After I/R, immunoreactivity for both iNOS and nNOS was detected, mainly in the interstitial space around damaged tubules, while germ cells in the damaged tubules were immunostained intensely for eNOS. We postulate that increased expression of the three NOS isoforms in the testis after $\mathrm{I} / \mathrm{R}$, which might generate nitric oxide, affects delayed germ cell death following $\mathrm{I} / \mathrm{R}$ via paracrine or autocrine fashion.

KEY WORDS: ischemia/reperfusion, nitric oxide synthase, testis.

J. Vet. Med. Sci. 67(4): 453-456, 2005

Testicular torsion has been implicated in testicular injury and infertility. The main pathology of testicular torsion is ischemia and reperfusion (I/R) injury of the testis, which is caused by the twisting and subsequent release of the spermatic cord [18]. The reperfusion is one of the most important factors in further injury [1]. In this animal model, several signal transduction molecules are expressed in the death/survival of the testicular cells following I/R. Several recent studies have reported on the expression of cell death molecules, including Bax, caspase 1, 2, and 3, Fas, Fas ligand, and nitric oxide (NO) $[4,5,11]$ and survival molecules, including nerve growth factor and hepatocyte growth factor, and their receptors $[8,9]$ and their possible roles in germ cell death and survival following $\mathrm{I} / \mathrm{R}$ in the rat testis.

Although several investigators have reported that $\mathrm{NO}$, which has dual effects on cell survival and death, is involved in testicular disorders, including testicular torsion and inflammation $[2,11,12,19]$, little is known about the functional roles and temporal profiles of the synthases, including the constitutive and inducible forms of NO synthase (NOS), in acute testicular I/R. Nevertheless, the critical roles of NO generated by the three NOS isoforms-inducible NOS (iNOS), endothelial NOS (eNOS), and neuronal NOS (nNOS) - in cell growth, differentiation, and death have been delineated.

This study examined the expression and localization of the three NOS isoforms (iNOS, eNOS, and nNOS) to investigate the possible role of NOS in the pathogenesis of testicular I/R injury in acute experimental testicular torsion.

Male adult Sprague Dawley rats (250-300 g) were anesthetized for surgery with sodium pentobarbital $(50 \mathrm{mg} / \mathrm{kg}$ body weight, i.p.). All surgical procedures were performed as mentioned in our previous studies [7-9]. The left scro-

\footnotetext{
* Correspondence to: Shin, T., Department of Veterinary Medicine, Cheju National University, Jeju 690-756, South Korea.
}

tum was incised, and the left spermatic cord was rotated $720^{\circ}$ clockwise to minimize individual variation in blood flow. After $1.5 \mathrm{hr}$, the torsion (i.e., ischemia) was relieved (i.e., reperfusion), and the testis was returned to the scrotum. Testes were collected at 3, 6, 12, 24, 48, and $96 \mathrm{hr}$ after reperfusion. Tissue samples were processed for paraffin embedding or stored at $-70^{\circ} \mathrm{C}$ for later biochemical analysis. All animal experiments followed a protocol approved by the Committee for Animal Experimentation, of the University of the Ryukyus.

Frozen tissues were thawed at room temperature, minced, homogenized, and lysed in a buffer containing $40 \mathrm{mM}$ Tris$\mathrm{HCl}$, pH 7.4, $120 \mathrm{mM} \mathrm{NaCl}$, and $0.1 \%$ Nonidet P-40 (polyoxyethylene [9] p-t-octyl phenol) supplemented with the protease inhibitors leupeptin $(0.5 \mu \mathrm{g} / \mathrm{m} l)$, PMSF $(1 \mathrm{mM})$, and aprotinin $(5 \mu \mathrm{g} / \mathrm{m} l)$. Equal amounts of protein $(200 \mu \mathrm{g} /$ $20 \mu l$ ) were loaded in each lane and electrophoresed under denaturing conditions in sodium dodecyl sulfate-polyacrylamide gels (SDS-PAGE). After electrophoresis, the proteins were electrotransferred onto nitrocellulose transfer membranes (Schleicher and Schuell, Keene, NH). Blotting with rabbit anti-iNOS antisera, mouse anti-eNOS antibodies, or rabbit anti-nNOS antisera (1:1,000 dilution, Transduction Laboratories, Lexington, KY) was performed as described in our previous study [9]. Subsequently, the bound antibodies were stripped from the membranes, and the membranes were reprobed with monoclonal antibody to beta actin (Sigma, St. Louis, MO). The density of each band was measured using a scanning densitometer (GS-700, Bio-Rad Laboratories, Hercules, CA) and analyzed using Molecular Analyst software (Bio-Rad). The ratios of each of the three isoforms of NOS to beta actin were compared using oneway analysis of variance (ANOVA), followed by a Newman-Keuls post hoc test. In all cases, values of $p<0.05$ were considered statistically significant.

Immunohistochemical staining was performed, with 
slight modifications, in the manner of our previous study [9]. In brief, 5- $\mu \mathrm{m}$ sections of paraffin-embedded testis were deparaffinized and incubated with optimally diluted primary antisera, including rabbit anti-iNOS, rabbit antinNOS, or mouse anti-eNOS antisera (Transduction Laboratories, Lexington, KY). The immunoreactions were visualized using avidin-biotin peroxidase complexes (Elite kit; Vector, Burlingame, CA), and the peroxidase reaction was developed using a diaminobenzidine substrate kit (Vector).

As seen in Fig. 1, iNOS expression was increased remarkably at $24 \mathrm{hr}$ after reperfusion (lane 5, $82.7 \pm 18.4$ $[$ mean $\pm \mathrm{SEM}]$ fold, $\mathrm{n}=4, p<0.05)$, and there was a significant peak at $48 \mathrm{hr}$ (lane $6,156.8 \pm 39.1$ fold, $\mathrm{n}=4, p<0.001$ ), compared with the normal controls (lane 1). Thereafter, iNOS expression declined slightly by $96 \mathrm{hr}$ (lane 7, $117.7 \pm$ 30.5 fold, $\mathrm{n}=3, p<0.05$ vs. the normal controls). Moreover, a significant increase in eNOS expression began at $24 \mathrm{hr}$ after reperfusion (lane 5, $2.07 \pm 0.37$ fold, $n=4, p<0.05$ ), and showed a significant peak at $48 \mathrm{hr}$ (lane 6, $2.01 \pm 0.64$ fold, $\mathrm{n}=4, p<0.05$ vs. the normal controls). After reperfusion, the expression of nNOS showed a pattern similar to that of eNOS from 24 (lane 5, $2.17 \pm 0.32$ fold, $\mathrm{n}=4, p<0.05 \mathrm{vs}$. the normal controls) to $48 \mathrm{hr}$ (lane $6,2.76 \pm 0.14$ fold, $\mathrm{n}=4$, $p<0.01$ vs. the normal controls).

iNOS immunoreactivity was rarely found in the normal rat testis (Fig. 2A), while its immunoreactivity was significantly increased at $48 \mathrm{hr}$ after I/R, mainly in the interstitial space (inflammatory cells, Leydig cells, and vessels) surrounding the damaged seminiferous tubules (Fig. 2B). Constitutive expression of eNOS was noted mainly in vessels of the testes of normal controls (Fig. 2C). At $48 \mathrm{hr}$ after reperfusion, eNOS immunoreactivity was greatly increased in the majority of germ cells in damaged tubules, as well as in vessels (Fig. 2D). By contrast, nNOS was occasionally detected only in the interstitial space of the rat testis (Fig. 2E). nNOS immunoreactivity was obviously enhanced, mainly in the interstitial cells, most likely Leydig cells, and round monocyte-like inflammatory cells around damaged tubules at $48 \mathrm{hr}$ after repairing the torsion (Fig. 2F).

This study demonstrated that both constitutive eNOS and nNOS are increased significantly at $24-48 \mathrm{hr}$ after I/R, using a semi-quantitative analysis. These synthases might be involved in the delayed phase in acute experimental testicular torsion. To examine the relationship between NOS expression and delayed germ cell death, we analyzed the immunostaining pattern of the three isoforms of NOS in the
A

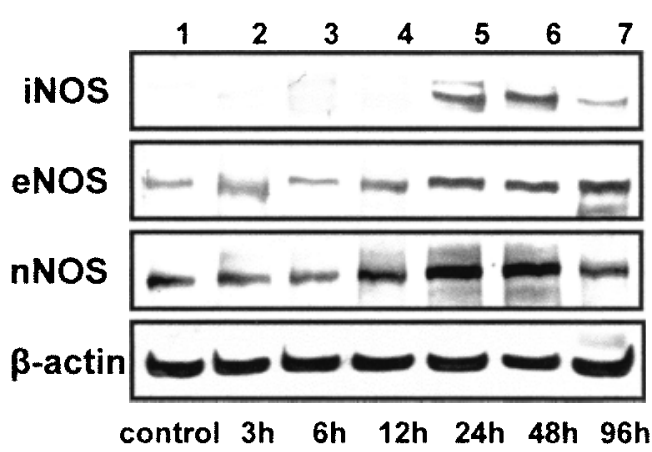

C

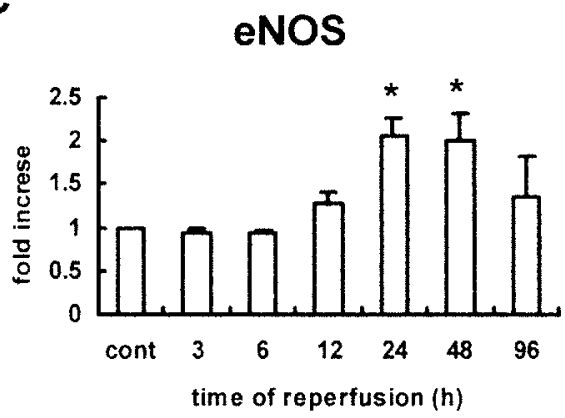

B

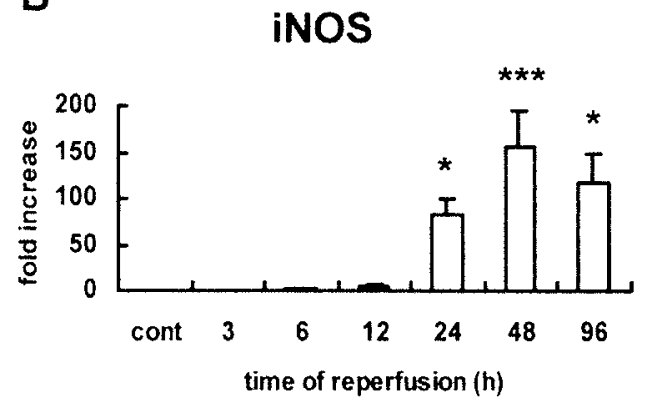

D

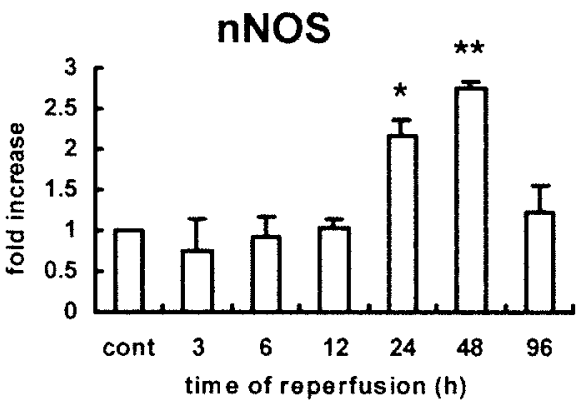

Fig. 1. Immunoblots of iNOS, eNOS, and nNOS in normal control rat testes (lane 1) and rat testes at 3 (lane 2), 6 (lane 3), 12 (lane 4), 24 (lane 5), 48 (lane 6), and $96 \mathrm{hr}$ (lane 7) after reperfusion following $1.5 \mathrm{hr}$ of experimental testicular torsion. A, Representative Western blots of iNOS (140 kDa), eNOS (130 kDa), nNOS (155 $\mathrm{kDa})$, and beta-actin $(45 \mathrm{kDa})$ in the testis with $\mathrm{I} / \mathrm{R}$. B-D, The amount of protein in each band was quantified using a densitometer. The value in each figure is the fold increase relative to the density in the normal control (cont) after normalization to beta actin. The relative expression of iNOS (B), eNOS (C), and nNOS (D) was calculated, and the value for the normal control was arbitrarily defined as $1 . *, p<0.05, * *, p<0.01$ and ***, $p<0.001$ vs. normal controls. 
Control

\section{iNOS}

\section{eNOS}
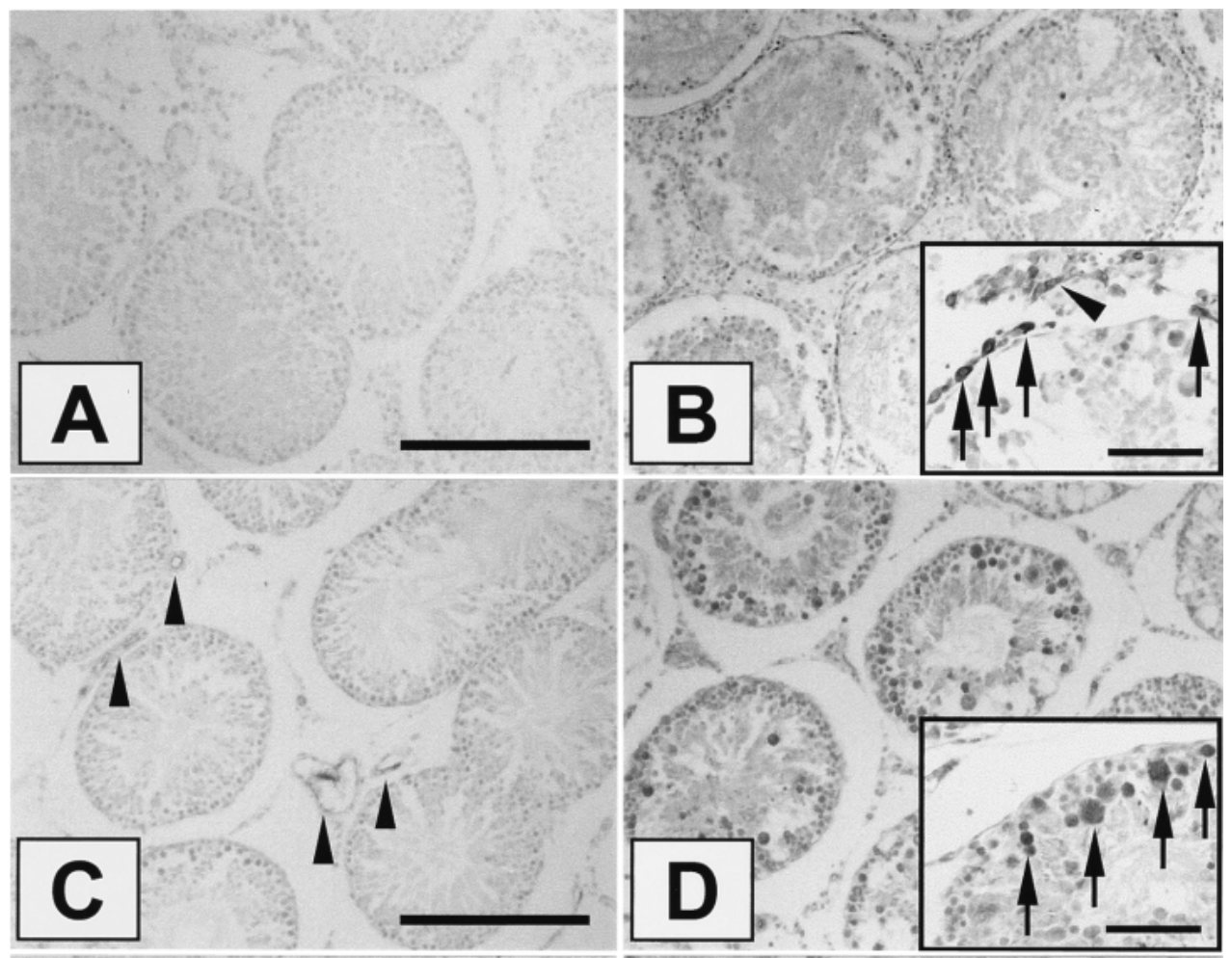

nNOS
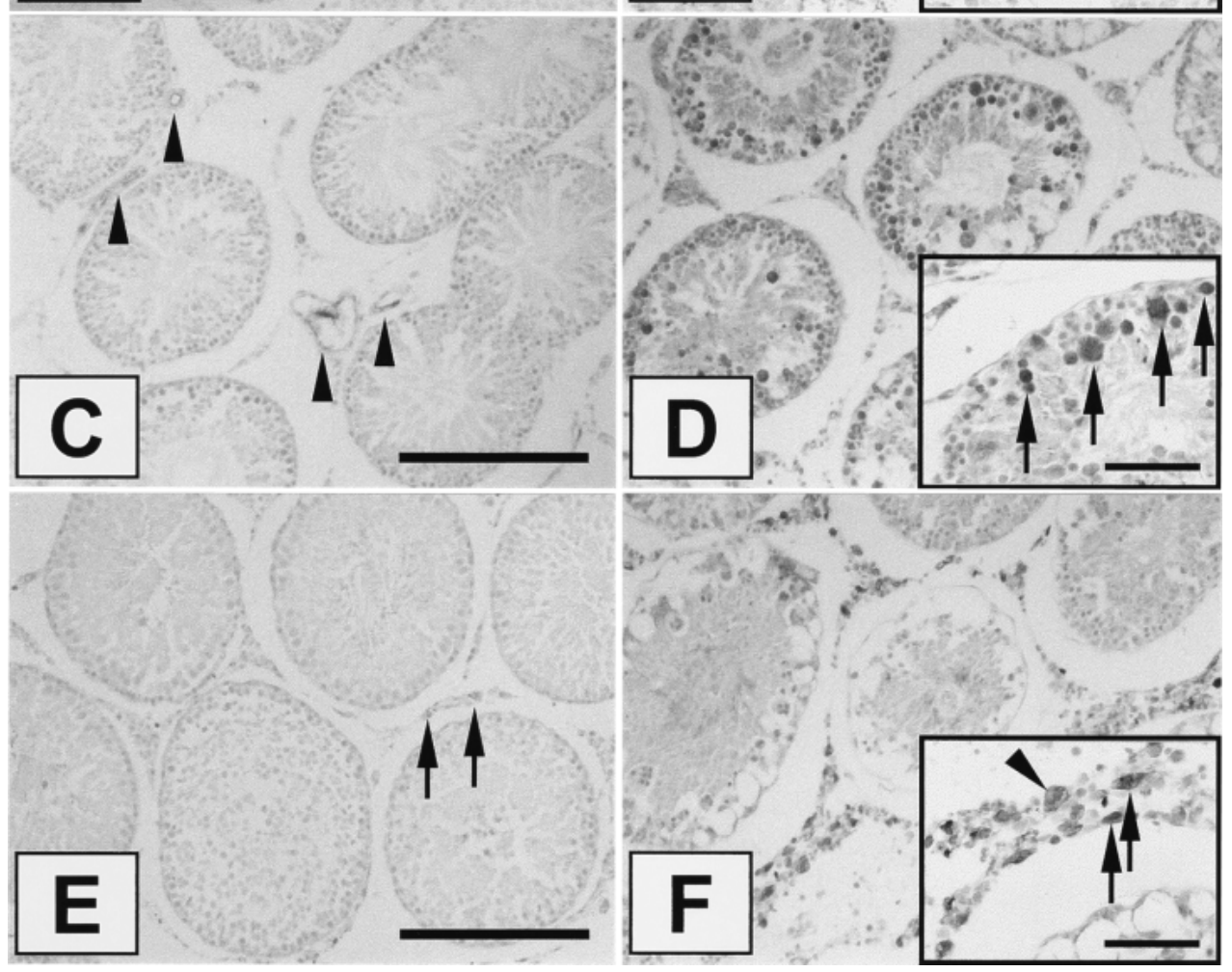

Fig. 2. Immunostaining of iNOS, eNOS, and nNOS in the testes of normal rats and rats with testicular torsion. A, C, and E: Normal control testes. B, D, and F: Rat testis at $48 \mathrm{hr}$ after relieving the torsion. iNOS immunoreactivity was rarely seen in the normal control (A); after I/R, iNOS immunoreactivity was obviously increased in inflammatory cells (B, inset, arrows) and in Leydig cells (B, inset, arrowhead) surrounding the damaged tubules. In normal controls, constitutive eNOS expression was noted mainly in the vessels of the testes (C, arrowheads). After I/R, eNOS expression was markedly increased in the germ cells in damaged tubules (D, inset, arrows), as well as in the vessels (D, arrowheads). nNOS was occasionally detected only in the interstitial space of the normal rat testis (E, arrows), while its expression was obviously enhanced around damaged tubules following I/R, mainly in the interstitial cells (most probably Leydig cells; F, inset, arrows) and macrophages (F, inset, arrowhead). Counterstained with hematoxylin. Scale bar $=200 \mu \mathrm{m}$; scale bar in inset $=50 \mu \mathrm{m}$.

testis with $\mathrm{I} / \mathrm{R}$.

$\mathrm{NO}$ is a free radical synthesized by NOS, including constitutive eNOS and nNOS, and iNOS. The first two isoforms constitutively generate a physiological level of NO (picograms) and are involved in neurotransmission and vasorelaxation, while iNOS produces a large amount of NO (nanograms) and provides important bactericidal, tumoricidal, and cytotoxic actions $[6,10,14]$. Although constitutively generated NO acts as an intracellular and/or extracellular messenger, it can be toxic to cells at high con- 
centrations, owing to the inhibition of DNA replication and lipid peroxidation.

Germ cell apoptosis peaks at $24 \mathrm{hr}$ after testicular torsion and reperfusion, and declines by $48 \mathrm{hr}$ after reperfusion [79]. The first signs of germ cell necrosis appeared at $24 \mathrm{hr}$ after I/R in the present study and gradually increased thereafter. This sequential appearance of programmed cell death and necrosis may be attributable to the induction of apoptosis by low concentrations of NO, followed by the induction of necrosis by high concentrations of $\mathrm{NO}[5,7,9,17]$.

Our results generally agree with those of a previous study [14] that indicated that iNOS increases markedly during the delayed phase ( 24 to $96 \mathrm{hr}$ after reperfusion) of testicular torsion and that the inhibition of NOS ameliorates the incidence of testicular necrosis, but fails to reduce apoptosis $[14,19]$. This suggests that excessive NO produced by iNOS around damaged tubules (Fig. 2) participates in the mediation of delayed germ cell necrosis in a paracrine fashion.

Moreover, the pattern of delayed cell necrosis largely matched the pattern of expression of eNOS in this study; eNOS expression was significantly increased in damaged germ cells at 24-48 hr after I/R. This finding contrasts with a previous study showing that eNOS immunoreactivity in some TUNEL- (in situ nick end-labeling) positive germ cells during the early phase after testicular torsion related to germ cell-specific apoptosis following I/R [19], rather than to delayed cell necrosis. However, we found that germ cells in the damaged tubules were increasingly immunopositive for eNOS in the delayed phase after I/R (Fig. 2), which suggests that increasing amounts of NO produced in damaged tubules by eNOS as well as iNOS affect the degeneration of germ cells by autocrine or paracrine means following I/R (apoptosis in the early phase and necrosis in the delayed phase after $\mathrm{I} / \mathrm{R})$.

It is generally accepted that nNOS also functions in nonneuronal tissues. Previous studies have shown that testosterone-producing Leydig cells are positive for nNOS in human [16] and horse testes [3], and have suggested that nNOS plays a modulatory role in the biology of interstitial cells that produce testosterone, as well as in spermatogenesis in the seminiferous tubules. Moreover, a previous study suggested that nNOS expression in inflammatory hematogenous cells, including macrophages, may play an important role in the pathogenesis of CNS inflammation [13]. In this study, we observed that the expression of nNOS in the testis was significantly increased in the delayed phase after testicular torsion. Although the pattern of immunostaining for nNOS was similar to that for iNOS in the testis with testicular torsion, intense immunoreactivity for nNOS was seen mainly in interstitial cells, most probably Leydig cells, rather than in inflammatory cells.

In addition, we found differential expression patterns for the three isoforms of NOS in the testis with I/R. The expression of both iNOS and nNOS was seen in the interstitial cells, most probably Leydig cells, and in infiltrating macrophages around damaged tubules, while eNOS expression was localized to the germ cells of severely damaged seminiferous tubules in which germ cell necrosis was also evident. These findings suggest that eNOS and nNOS are also involved in the destruction of tissue owing to NO in acute experimental testicular torsion, in a paracrine or autocrine fashion, although the functional roles of eNOS and nNOS in germ cells, interstitial cells (most probably Leydig cells), and infiltrating inflammatory cells in testicular disease remain unclear.

In conclusion, the three isoforms of NOS appear to be involved in the pathogenesis of testicular I/R injury. Specifically, increased expression of the three isoforms in the delayed phase after I/R seems to relate to delayed germ cell death (especially necrosis) following $\mathrm{I} / \mathrm{R}$.

ACKNOWLEDGMENT. This study was supported by grant No. R01-2002-000-00053-0 (2004) from the Basic Research Program of the Korean Science \& Engineering Foundation.

\section{REFERENCES}

1. Akgur, F.M., Kilinc, K. and Aktuğ, T. 1993. Urol. Res. 21: 395-399.

2. Barlas, M. and Hatiboglu, C. 2002. Int. Urol. Nephrol. 34: 8186.

3. Ha, T.Y., Kim, H.S. and Shin, T. 2004. J. Vet. Med. Sci. 66: 351-356.

4. Koji, T. 2001. Med. Electron Microsc. 34: 213-222.

5. Lysiak, J.J., Turner, S.D. and Turner, T.T. 2000. Biol. Reprod. 63: $1465-1472$.

6. Moncada, S., Palmer, R.M. and Higgs, E.A. 1991. Pharmacol. Rev. 43: 109-142.

7. Moon, C. and Shin, T. 2004. Korean J. Vet. Res. 44: (in press).

8. Moon, C., Yasuzumi, F., Kim, H., Ahn, M., Sekiguchi, K., Okura, N. and Shin, T. 2004. Anat. Sci. Int. 79 (Suppl.): 257.

9. Moon, C., Yasuzumi, F., Okura, N., Kim, H., Ahn, M. and Shin, T. 2004. Urol. Int. (in press).

10. Nathan, C. and Xie, Q.W. 1994. J. Biol. Chem. 269: 1372513728.

11. Ozokutan, B.H., Kucukaydin, M., Muhtaroglu, S. and Tekin, Y. 2000. J. Pediatr. Surg. 35: 101-103.

12. Ozturk, H., Buyukbayram, H., Ozdemir, E., Ketani, A., Gurel, A., Onen, A. and Otcu, S. 2003. J. Pediatr. Surg. 38: 16211627.

13. Shin, T., Weinstock, D., Castro, M.D., Acland, H., Walter, M., Kim, H.Y. and Purchase, H.G. 2000. J. Vet. Sci. 1: 77-80.

14. Shiraishi, K., Naito, K. and Yoshida, K. 2001. Biol. Reprod. 65: $514-521$.

15. Skaper, S.D., Facci, L. and Leon, A. 1995. J. Neurochem. 64: 266-276.

16. Wang, Y., Newton, D.C., Miller, T.L., Teichert, A.M., Phillips, M.J., Davidoff, M.S. and Marsden, P.A. 2002. Am. J. Pathol. 160: $369-380$.

17. Weins, S.W. and Lucchesi, B.R. 1990. Trends Physiol. Sci. 11: 161-167.

18. Williamson, R.C.N. 1977. New Engl. J. Med. 296: 338.

19. Zini, A., Abitbol, J., Girardi, S.K., Schulsinger, D., Goldstein, M. and Schlegel, P.N. 1998. Arch. Androl. 41: 57-65. 